\title{
The Extended Malkus-Robbins Dynamo as a Perturbed Lorenz System
}

\author{
IRENE M. MOROZ \\ Mathematical Institute, 24-29 St Giles', Oxford OX1 3LB, UK (e-mail: moroz@maths.ox.ac.uk; fax: +44-1865-270515)
}

(Received: 24 February 2004; accepted: 10 March 2004)

\begin{abstract}
Recent investigations of some self-exciting Faraday-disk homopolar dynamos [Hide, R. and Moroz, I. M., Physica D 134, 1999, 387-301; Moroz, I. M. and Hide, R., International Journal of Bifurcation and Chaos 2000, 2701-2716; Moroz, I. M., International Journal of Bifurcation and Chaos 13, 2003, 147-161; Moroz, I. M., International Journal of Bifurcation and Chaos, to appear] have yielded the classic Lorenz equations as a special limit when one of the principal bifurcation parameters is zero. In this paper we focus upon one of those models [Moroz, I. M., International Journal of Bifurcation and Chaos 13, 2003, 147-161] and illustrate what happens to some of the lowest order unstable periodic orbits as this parameter is increased from zero.
\end{abstract}

Key words: dynamos, Lorenz equations, unstable periodic orbits

\section{Introduction}

Self-exciting dynamos are nonlinear electro-mechanical engineering devices or naturally occurring magnetohydrodynamic (MHD) fluid systems such as the geodynamo, operating within the Earth's liquid metallic outer core, that convert mechanical energy into magnetic energy through the action of motional induction without involving permanent magnets. Because of the intractablity of the governing nonlinear partial differential equations in four independent variables, low-dimensional analogues, capable of providing valuable insights into the on-going physical processes have been proposed and investigated.

In a seminal paper Hide et al. [5] introduced one such system, comprising three coupled nonlinear ordinary differential equations, to model the behaviour of a self-exciting homopolar Faraday disk dynamo, in an effort to increase our understanding of phenomena such as self-sustaining dynamo action in the Earth's liquid core, especially as regards geomagnetic polarity reversals, and the El-Niño Southern Oscillation, which plays such a dramatic role in influencing global weather patterns.

Later studies extended the original model of [5] to include investigations of the effects due to a nonlinear series motor [6]; coupled systems of Faraday disk dynamos [7, 8]; effects due to azimuthal eddy currents in a single disk dynamo [1,2]; the identification of conditions for phase- and amplitudelocked states in coupled disk dynamos with linear series motors $[7,9,10]$ and the effects due to external battery and magnetic field terms on the behaviour of a single disk dynamo [11, 12].

There has been considerable interest in recent years in identifying the spectrum of underlying unstable periodic orbits (or upos), associated with chaotic time series, as a way of characterising the attractors of nonlinear dynamical systems such as the Lorenz equations (see, for example, [13, 14]), themselves the lowest order truncation of the partial differential equations governing Benard convection. As noted by Carroll [14], while a chaotic attractor possesses an infinite number of upos, many properties may be determined from those orbits of lowest period. 
Recent investigations of several different self-exciting Faraday-disk dynamos [1-4] have shown the classic Lorenz equations to result in a special limit when one of the key bifurcation parameters, $\beta$, which measures the inverse moment of inertia of the armature of the dynamo motor, vanishes. In this paper we investigate one of those models [3], which we term the extended Malkus-Robbins dynamo (or EMR), since it reduces to the Malkus-Robbins equations [15] when $\beta=0$. The other dynamo models will form the subjects of future studies. The EMR dynamo, as well as the other two dynamos which have the Lorenz equations as a limiting case, is a set of four coupled nonlinear ordinary differential equations, which results from the removal of two of the three electric motors in one of the dynamo models introduced by Hide [8] and their replacement by resistances.

When $\beta=0$, one of the equations decouples, leaving the remaining three as the Lorenz equations, after a simple linear transformation. An exposition of the derivation of the EMR system, its linear stability analysis and bifurcation transition sequences can be found in [3]. Our current objectives are to illustrate what happens to the lowest order upos, as computed via numerical integrations, as well as the return maps as we move away from the Lorenz limit.

Following Koga [13], we have labelled each upo as coming from series $L_{n m}$, where $n$ denotes the number of circuits around the left non-trivial equilibrium state, while $m$ the number of circuits around the right non-trivial equilibrium state. In our investigations we have considered $n, m=1, \ldots, 10$. Since any particular initial data set may not yield all of the possible upos up to a certain order, we have proceeded as follows. We used the same initial conditions for each numerical integration, plotting histograms up to, and including, $n=m=10$, for all of those orbits which returned to within a given distance $\epsilon$ on the Poincaré section in four-dimensional phase space.

Following Koga [13], we transformed the EMR equations so that this Poincare section is on the equivalent to the $Z=r-1$ plane for the original Lorenz equations (see Section 3). Moreover, we have used the classic Lorenz parameters of $r=28, b=8 / 3$ and $\sigma=10$ throughout, indicating what these correspond to for the dynamo model.

This paper is structured as follows. In Section 2, we briefly review the pertinent features of calculating the upos and return maps for the Lorenz equations as described in [13]. Section 3 introduces the EMR model and its transformation which was used in the upo study. We present two cases, including linear stability curves in regime diagrams, as well as bifurcation transition sequences for the classic Lorenz parameter values, as $\beta$ is increased from zero until the loss of the chaotic regime. In Section 4 we present the return maps and histograms at selected values of $\beta$. Finally we draw our conclusions in Section 5 .

\section{Unstable Periodic Orbits in the Lorenz Equations}

In this section we shall summarise those aspects of Koga's treatment of the Lorenz equations that will serve as a comparison for the dynamo model of the next two sections.

The Lorenz equations

$$
\begin{aligned}
& \dot{x}=\sigma(y-x), \\
& \dot{y}=r x-y-x z, \\
& \dot{z}=-b z+x y,
\end{aligned}
$$

possess three equilibrium solutions

$$
\left(x_{0}, y_{0}, z_{0}\right)=(0,0,0), \quad\left(x_{\mathrm{e}}, y_{\mathrm{e}}, z_{\mathrm{e}}\right)=( \pm \sqrt{b(r-1)}, \pm \sqrt{b(r-1)}, r-1) .
$$


The change of variable

$$
\hat{z}=z-(r-1)
$$

transforms (1)-(3) to

$$
\begin{aligned}
& \dot{x}=\sigma(y-x), \\
& \dot{y}=-y+x(1-\hat{z}), \\
& \dot{\hat{z}}=-b(\hat{z}+r-1)+x y,
\end{aligned}
$$

and the equilibria to

$$
\left(x_{0}, y_{0}, \hat{z}_{0}\right)=(0,0,1-r), \quad\left(x_{\mathrm{e}}, y_{\mathrm{e}}, \hat{z}_{\mathrm{e}}\right)=( \pm \sqrt{b(r-1)}, \pm \sqrt{b(r-1)}, 0) .
$$

Taking the Poincaré section to be

$$
S=[(x, y): \hat{z}=0, \dot{\hat{z}}>0, x>0],
$$

Koga is able to construct two one-dimensional return maps

$$
X_{n+1}=f_{1}\left(X_{n}\right), \quad Y_{n+1}=f_{2}\left(Y_{n}\right),
$$

the fixed points of which correspond to the upos of the series $L_{n 1}$ for $n=1,2, \ldots$ Here $L_{n m}$ refers to the trajectory rotating $n$ times around the left focus and $m$ times the right focus, where the foci are the nontrivial equilibrium solutions (8) of the Lorenz equations. The upos associated with $L_{n m}$ for $m>1$, are generated by iterating the return maps $m$ times and finding their fixed points.

In this paper we present results for the classic Lorenz parameter choices of $r=28, \sigma=10$ and $b=8 / 3$. Figure 1 reproduces Figure 2 of [13] and shows the first return map in the $\left(X_{n}, X_{n+1}\right)$-plane for which $\hat{z}=0$, corresponding to $z=27$. The intersections of the arcs of dots with the straight line $X_{n}=X_{n+1}$ give the $L_{11}, L_{21}, \ldots$ in ascending order. To ensure an accurate computation of the values of $\left(X_{n}, Y_{n}\right)$ on the Poincaré section, we incorporated the numerical code suggested by Hénon [16]. In our numerical studies, we integrated the equations for $60,000 \mathrm{~s}$ using a time step of $0.001 \mathrm{~s}$, discarding the first $100 \mathrm{~s}$ as representing transients. Figure 1 contains 39,678 points.

Figure 2 shows the histogram of periods of the orbits, as functions of the number of close returns within a ball of radius $\epsilon=0.05$. In terms of the upos $L_{n m}$, they represent close returns to within $\epsilon=0.05$ to the fixed points of the $m$ th iterates of the return map for $m=1, \ldots, 10$. Comparisons of our results with those of $[13,17,18]$ were made to validate the accuracy of our approach in calculating the periods of the upos.

We end this section by remarking that upos for which the periods differ slightly from the ones tabulated in [13] correspond to orbits which differ slightly in the order in which they circuit around the left or the right equilibrium state. 


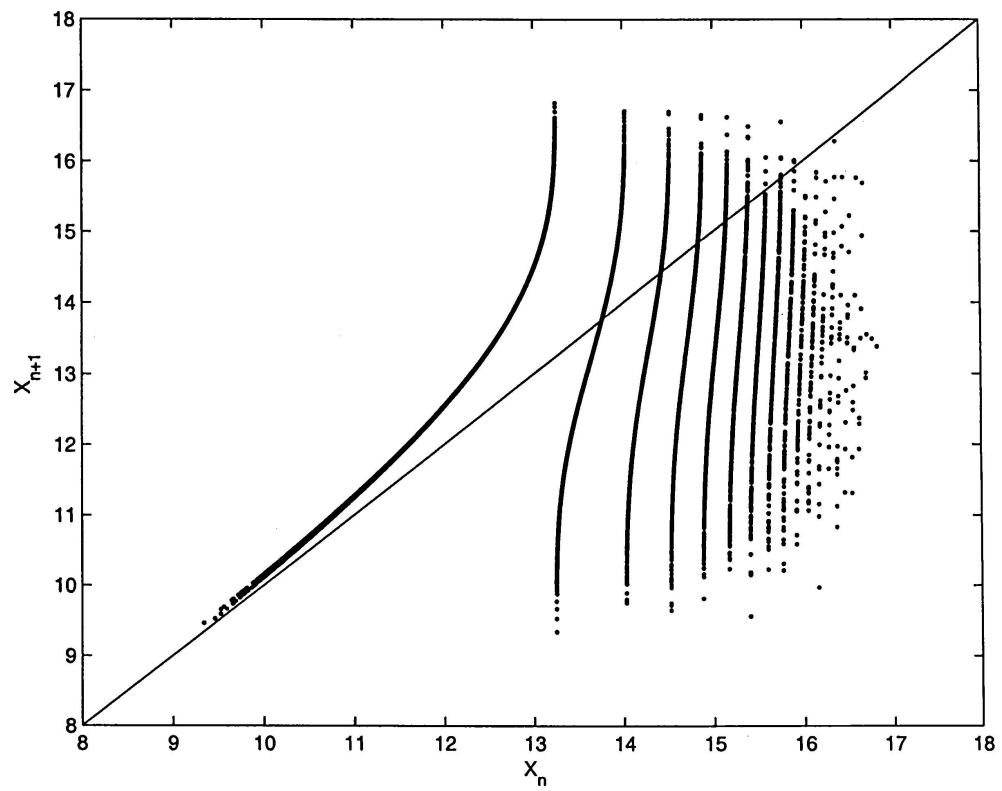

Figure 1. The first return map in the $\left(X_{n}, X_{n+1}\right)$-plane for the Lorenz equations.

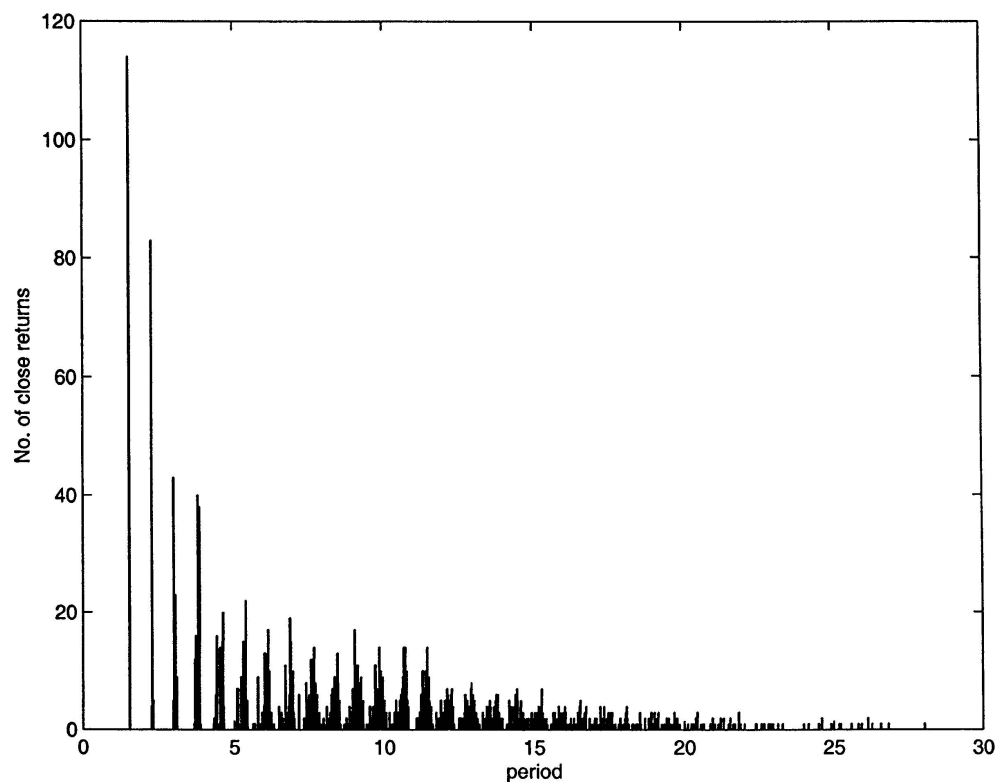

Figure 2. The histogram of periods for close returns for the first 10 iterates of the Lorenz first return maps.

\section{The Extended Malkus-Robbins Dynamo Equations}

\subsection{THE LORENZ LIMIT}

The nondirectional extended Malkus-Robbins (EMR) dynamo

$$
Y^{\prime}=\sigma(Z-Y)-\hat{\beta} U,
$$




$$
\begin{aligned}
Z^{\prime} & =Y W-Z, \\
W^{\prime} & =R-\alpha Z Y-v W, \\
U^{\prime} & =Y-\Lambda U,
\end{aligned}
$$

is a system of four coupled nonlinear differential equations, describing the behaviour of the MalkusRobbins dynamo [15], when a motor is coupled to the disk and coil. In (11)-(14) $Z(t)$ represents the current flowing through the disk; $Y(t)$ is the current flowing through the coil; $W(t)$ the angular velocity of the rotating disk and $U(t)$ the angular velocity of the motor.

The parameters appearing in (11)-(14) are all real and positive: $\alpha$ represents the steady couple, applied to the disk and causing it to rotate; $v$ is the re-scaled mechanical friction $\kappa$ of the disk and $\Lambda$ that of the motor; $R$ is a scaled and translated version of $\alpha ; \hat{\beta}=v^{2} \beta / \kappa^{2}$, where $\beta$ measures the inverse moment of inertia of the armature of the motor and $\sigma$ depends upon the inductances and resistances of the coil and brushes. The reader is referred to [3] for a more detailed discussion and derivation of (11)-(14).

It is possible to remove explicit reference to $\alpha$ from (13) via the transformation

$$
(W, Z, Y, U) \rightarrow \alpha^{-1 / 2}\left(\alpha^{1 / 2} W, Z, Y, U\right)
$$

If we also then introduce $\hat{W}=R / v-W,(3.1)$ becomes

$$
\begin{aligned}
Y^{\prime} & =\sigma(Z-Y)-\hat{\beta} U, \\
Z^{\prime} & =R / v Y-Y \hat{W}-Z, \\
\hat{W}^{\prime} & =Z Y-v \hat{W}, \\
U^{\prime} & =Y-\Lambda U .
\end{aligned}
$$

When $\hat{\beta}=0$, the evolution of $U$ effectively decouples from Equations (15)-(17) and we recover the Lorenz equations (1)-(3) under the correspondence

$$
(Y, Z, \hat{W}, U) \longmapsto(x, y, z, 0), \quad(\sigma, R / \nu, v, \hat{\beta}) \longmapsto(\sigma, r, b, 0) .
$$

The perturbations away from the Lorenz equations, which are the subject of this paper, involve the re-introduction of $\hat{\beta}$ and the investigation of what befalls the upos as $\hat{\beta}$ increases from zero.

\subsection{THE POINCARÉ SECTION}

The system (15)-(18) possesses three equilibrium solutions

$$
\mathbf{W}_{\mathbf{0}}=\left(Y_{0}, Z_{0}, \hat{W}_{0}, U_{0}\right)=(0,0,0,0)
$$

which we term the trivial equilibrium state and

$$
\mathbf{W}_{\mathrm{e}}=\left(Y_{\mathrm{e}}, Z_{\mathrm{e}}, \hat{W}_{\mathrm{e}}, U_{\mathrm{e}}\right),
$$


where

$$
\hat{W}_{\mathrm{e}}=\frac{R}{v}-\left(1+\frac{\hat{\beta}}{\Lambda \sigma}\right), \quad Z_{\mathrm{e}}=Y_{\mathrm{e}}\left(1+\frac{\hat{\beta}}{\Lambda \sigma}\right), \quad U_{\mathrm{e}}=\frac{Y_{\mathrm{e}}}{\Lambda}, \quad Y_{\mathrm{e}}= \pm \sqrt{\frac{v \hat{W}_{\mathrm{e}}}{\left(1+\frac{\hat{\beta}}{\Lambda \sigma}\right)}} .
$$

By analogy with the Lorenz equations, we take the Poincare section on $\mathcal{W}=0$ where

$$
\mathcal{W}=\hat{W}-\hat{W}_{\mathrm{e}}
$$

Then (15)-(18) transforms to

$$
\begin{aligned}
Y^{\prime} & =\sigma(Z-Y)-\hat{\beta} U, \\
Z^{\prime} & =Y\left[\left(1+\frac{\hat{\beta}}{\Lambda \sigma}\right)-\mathcal{W}\right]-Z, \\
\mathcal{W}^{\prime} & =Z Y-v\left(\mathcal{W}+\hat{W}_{\mathrm{e}}\right), \\
U^{\prime} & =Y-\Lambda U .
\end{aligned}
$$

The nontrivial equilibrium states are now

$$
\mathbf{W}_{\mathrm{e}}=\left(Y_{\mathrm{e}}, Z_{\mathrm{e}}, 0, U_{\mathrm{e}}\right)
$$

and the Poincaré section is

$$
\hat{S}=[(Y, Z, U): \mathcal{W}=0, \dot{\mathcal{W}}>0, Y>0]
$$

\subsection{The Linear Stability CURVES}

There are four different parameter regimes of interest, according as $\Lambda \gtrless 1$ and $\sigma \gtrless 1+v$ [3]. When $\Lambda<1$ and $\sigma>1+v$, as well as the Lorenz subcritical Hope bifurcation on $\hat{\beta}=0$, there is a Takes-Bodansky double-zero bifurcation in $\hat{\beta}>0$. A curve of subcritical Hope bifurcations connects the two bifurcation points. When $\Lambda>1$ and $\sigma>1+v$, no double-zero bifurcations are possible, and the curve of subcritical Hope bifurcations simply extends into the $\hat{\beta}>0$ regime. Since the Lorenz parameter values of $(r, \sigma, b)=(24.74,10,8 / 3)$ for the subcritical Hope bifurcation correspond to $(R, \sigma, v)=(65.965,10,8 / 3)$, we are in the $\sigma>1+v$ regime and do not need to investigate the remaining two cases outline above. The interested reader is referred to [3] for a discussion of these two cases within a different context. We remark that $r=28$ corresponds to $R=74.667$.

\section{4. $\Lambda>1$ AND $\sigma>1+v$}

As a typical example of what happens when $\Lambda>1$, we chose $\Lambda=3.2$ with $\sigma=10$ and $v=8 / 3$ throughout. Figure 3 shows the linear stability curves for the trivial and nontrivial equilibrium states. 


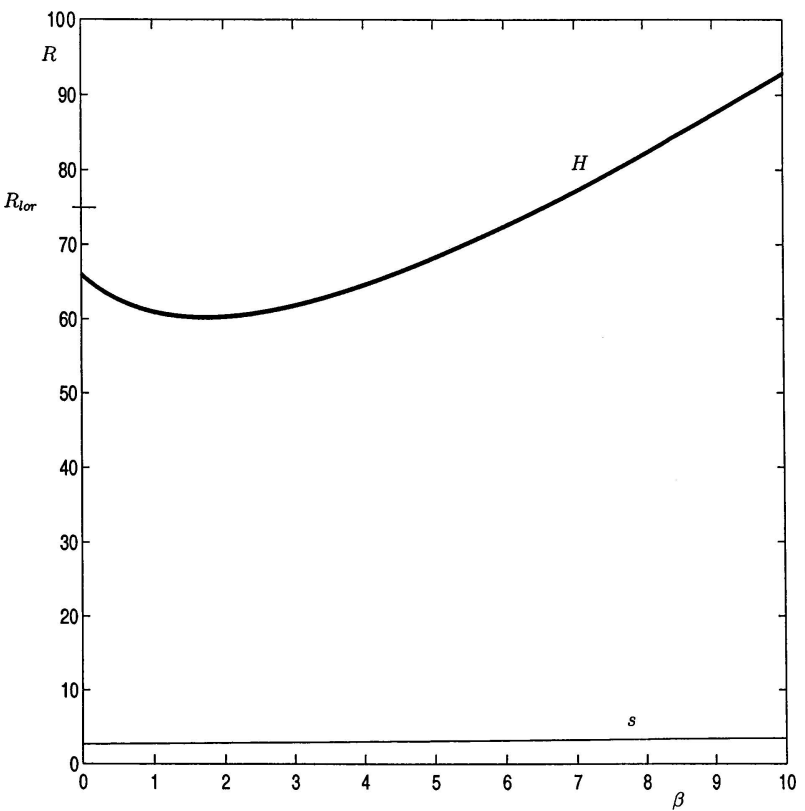

Figure 3. Linear stability curves for case (i) with $\Lambda=3.2, \sigma=10, v=8 / 3$ and $\kappa=1$. The Hope bifurcation curve associated with the nontrivial states is denoted by $H$, while the curve of steady bifurcation from the trivial equilibrium state is denoted by $s$. Also shown is the classic Lorenz parameter choice of $r=28$ or $R=74.667$.

There is a line of steady bifurcations (labelled as $s$ ) and given by

$$
R_{s}=v\left(1+\frac{\hat{\beta}}{\sigma \Lambda}\right),
$$

together with a curve of Hope bifurcations (labelled as $H$ ), which is computed numerically following the procedure outlined in [3]. We have used $\beta$ instead of $\hat{\beta}$, where $\hat{\beta}=\nu^{2} \beta / \kappa^{2}$, for ease of comparison with the EMR study in [3]. In all of our calculations we set $\kappa=1$.

As in the EMR study, steady dynamo solutions dominate the region between $H$ and $s$, with a narrow band of chaotic solutions extending slightly beyond $H$ because this is a curve of subcritical Hope bifurcations. The horizontal tick mark on the $R$ axis, and labelled $R_{\text {lor }}$, corresponds to $r=28$.

Figure 4 shows the bifurcation transition sequence observed as $\beta$ increases from zero for $r=28$. This is a plot of the local maximum amplitude of the oscillatory solution, as measured here by $\mathcal{W}$, as a function of $\beta$, having discarded the initial transient at each increment; the final conditions of one integration being used as the initial data for the next. Chaotic solutions extend well into the $\beta>0$ regime, until $\beta \approx 8$, when stability is lost to a steady state solution, given by the transformed equivalent to (22), which persists for larger values of $\beta$.

In Section 4 we shall see what happens to the maps of first return, the equivalent to the $\left(X_{n}, X_{n+1}\right)$ and/or $\left(Y_{n}, Y_{n+1}\right)$ maps for the Lorenz equations described in Section 2, as well as the histograms of upos as $\beta$ increases along this transition sequence.

\section{5. $\Lambda<1$ AND $\sigma>1+v$}

When $\Lambda=0.5$, we obtain the linear stability curves, shown in Figure 5. The curve $H$ of subcritical Hope bifurcations, associated with the nontrivial equilibrium solutions, now links the Lorenz subcritical 


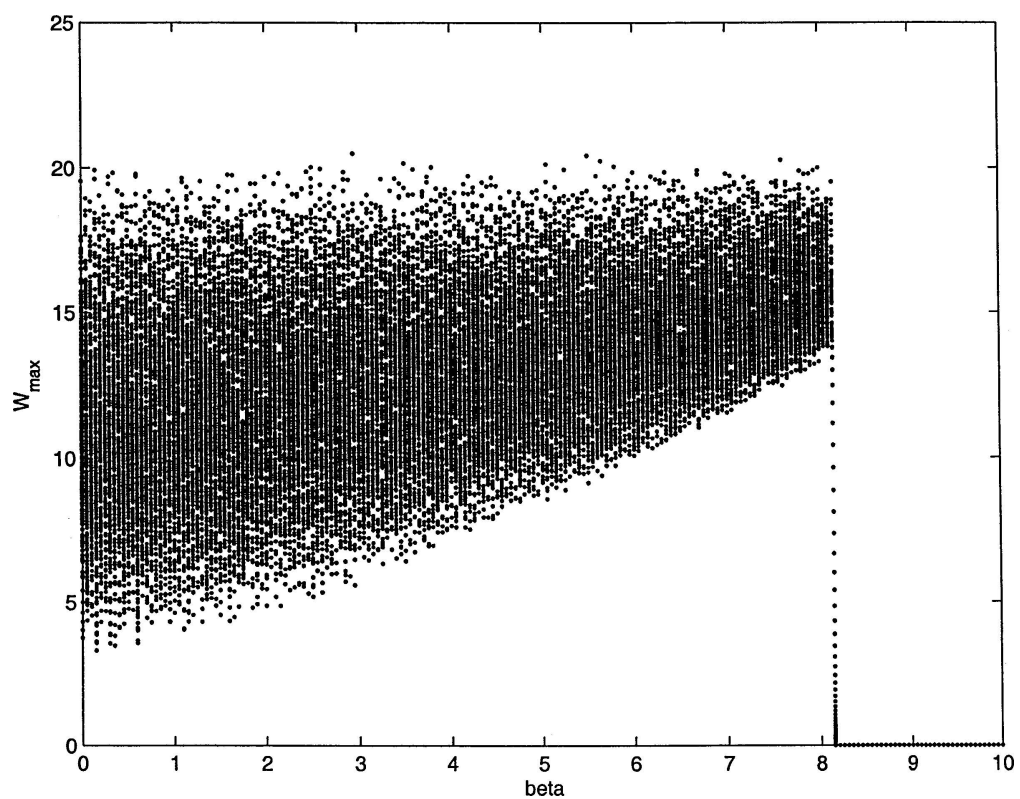

Figure 4. Plot of the local maximum values of $\mathcal{W}$ as a function of $\beta$ when $\beta$ is increased for $r=28$.

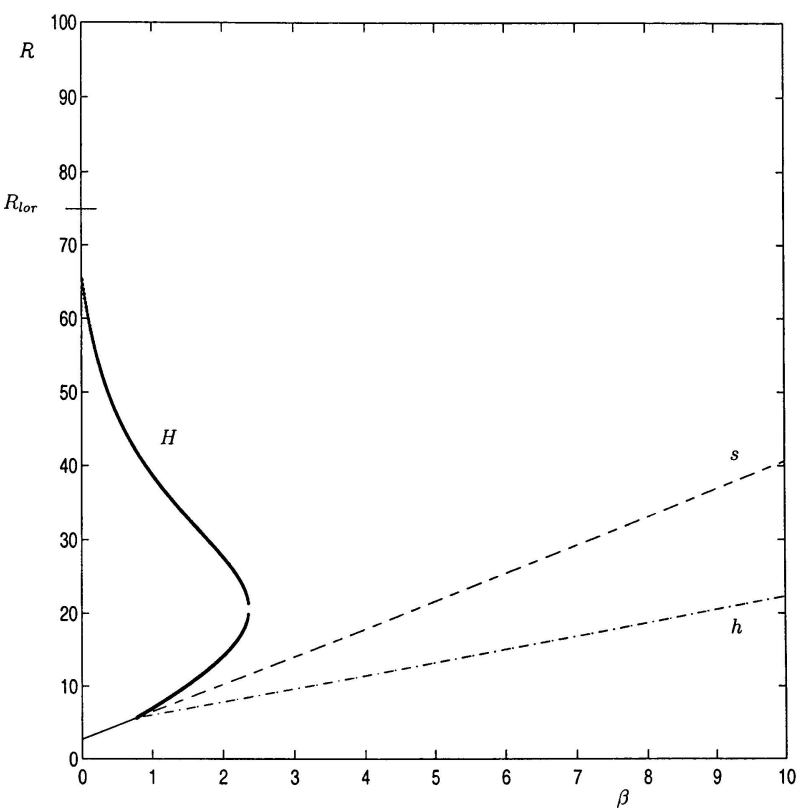

Figure 5. Linear stability curves for case (ii) with $\Lambda=0.5$, the remaining parameters are the same as for Figure 3 . As well as the curves $H$ and $s$, there is also a curve $h$ of Hope bifurcations from the trivial equilibrium state. The three curves $H, s$ and $h$ all meet at the double-zero point.

Hope bifurcation point on $\beta=0$ with the Takes-Bodansky bifurcation point in $\beta>0$, where all three curves $H, s$ and $h$ coincide. The curve $h$ is a curve of (supercritical) Hope bifurcations, associated with the trivial equilibrium solution.

Figure 6 shows the bifurcation transition sequence for $r=28$. The loss of chaotic behaviour is now to a stable periodic limit cycle when $\beta \approx 4$. 


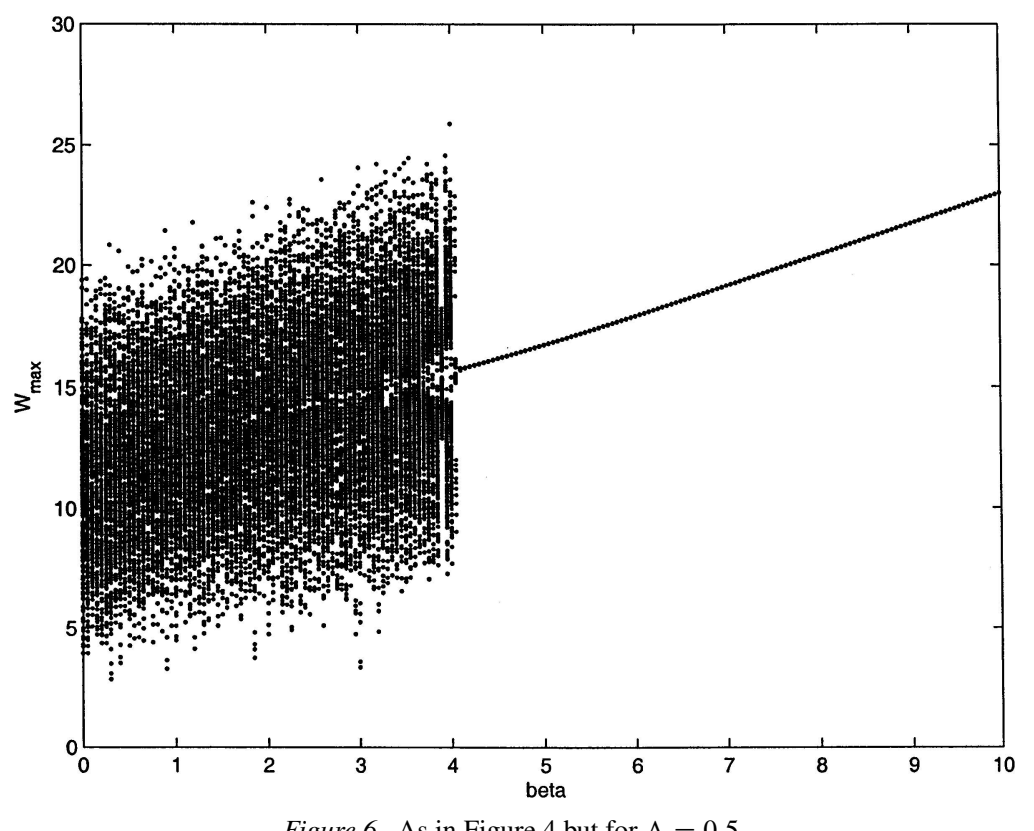

Figure 6. As in Figure 4 but for $\Lambda=0.5$.

\section{Upos in the EMR Dynamo}

We now present a selection of the results of our numerical integrations of (23)-(26) for $\beta \neq 0$ for the two cases outlined above. We preface this discussion with a comment concerning the Lorenz limit of (23)-(26) and the selection of a close return on the Poincaré section $\mathcal{W}=0$.

Since (23)-(26) is a four-variable system, we have used the condition

$$
\left\|\mathbf{W}_{i}-\mathbf{W}_{j}\right\|=\sqrt{\left(Y_{i}-Y_{j}\right)^{2}+\left(Z_{i}-Z_{j}\right)^{2}+\left(\mathcal{W}_{i}-\mathcal{W}_{j}\right)^{2}+\left(U_{i}-U_{j}\right)^{2}}<\epsilon
$$

which includes $U$ as opposed to

$$
\left\|\mathbf{X}_{i}-\mathbf{X}_{j}\right\|=\sqrt{\left(X_{i}-X_{j}\right)^{2}+\left(Y_{i}-Y_{j}\right)^{2}++\left(\hat{z}_{i}-\hat{z}_{j}\right)^{2}}<\epsilon
$$

for the Lorenz equations (5)-(7) to determine closeness of returns on $\mathcal{W}=0$, where $i$ and $j$ denote the $i$ th and $j$ th intersections. This has the consequence of producing a different number of close returns when $\beta=0$ than for the actual Lorenz equations, but yields identical first return maps.

In all of our numerical integrations we used the same initial conditions

$$
(Y, Z, \mathcal{W}, U)=(10.185360,5.8512615,9.4864804,2.5969457)
$$

to ensure that the variations in the histograms and in the first return maps were due solely to the variation in $\beta$, rather than reflecting any dependency upon the initial conditions. Our choice of $v=8 / 3$ means that $\hat{\beta}=7.1111 \beta$.

For the Lorenz equations, the Poincaré section $\hat{z}=0$ was equivalent to taking $z=27$ in (1)-(3). For the EMR equations, the Poincaré section $\mathcal{W}=0$ corresponds to taking $W=(1+\hat{\beta} / \Lambda \sigma)$ in (11)-(14). 

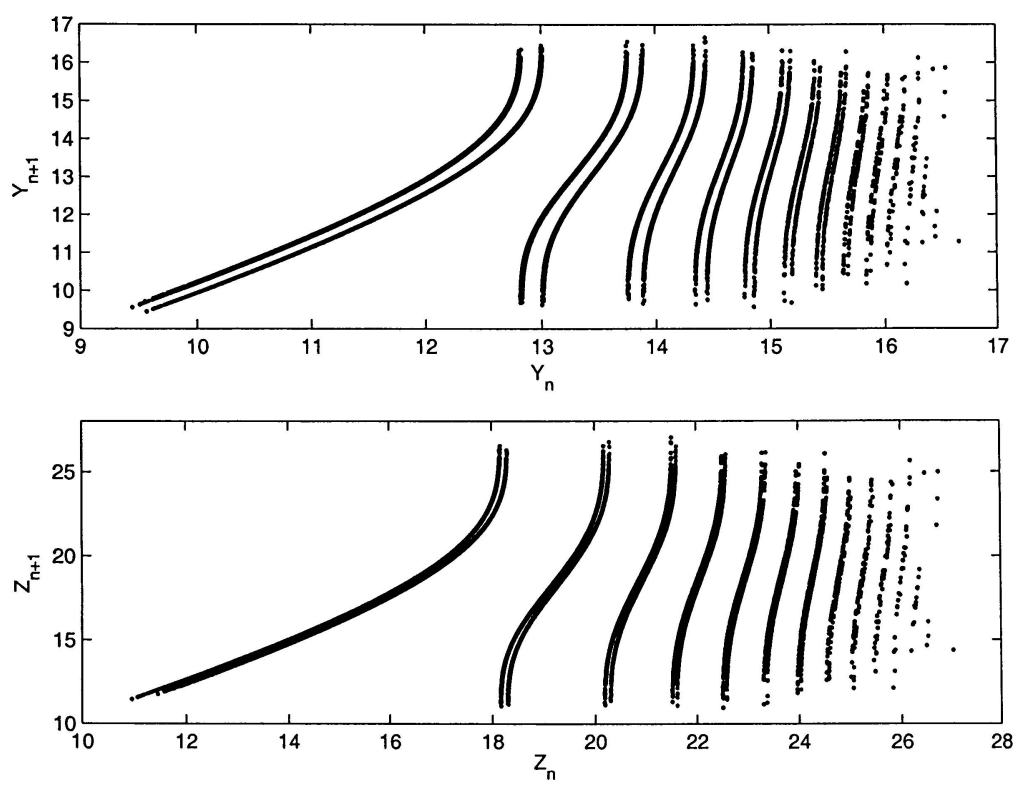

Figure 7. The first return maps of $Y_{n}$ and $Z_{n}$ for $\Lambda=3.2$ and $\beta=0.5$.

\section{1. $\Lambda>1 \operatorname{AND} \sigma>1+v$}

As in Section 2, we integrated the EMR equations (23)-(26) for 60, $000 \mathrm{~s}$ at a time step of $0.001 \mathrm{~s}$ (exactly the same as for the Lorenz equations), choosing $\epsilon=0.05$ as defining the ball of close returns. The maps of first returns are now plotted as $Y_{n} v Y_{n+1}$ and/or $Z_{n} v Z_{n+1}$ and histograms of the upos up to and including the 10th return maps are shown.

When $\beta=0$, the first return maps for $Y_{n}$ and $Z_{n}$ are identical to those for the Lorenz equations, and so are not reproduced here. We simply note that the histogram contains 504 close returns.

We now increase $\beta$. Figure 7 shows the $\left(Y_{n}, Y_{n+1}\right)$ and $\left(Z_{n}, Z_{n+1}\right)$ first returns for $\beta=0.5$, while Figure 8 shows the histogram up to series 10 . A very interesting banding structure has emerged in both maps, in marked contrast to the Lorenz limit, and which persists for higher values of $\beta$. Perusal of the close returns data shows the presence of $L_{11}$ (period of approximately $\left.1.562 \mathrm{~s}\right), L_{21}$ (2.324 s), $L_{31}(3.065$ s), $L_{41}(3.786 \mathrm{~s})$, and $L_{61}(5.226 \mathrm{~s})$ from the series $L_{n 1}$. The corresponding periods for these upos in the Lorenz system are [13]: $(1.5587,2.3059,3.0236,3.7256,5.1030) \mathrm{s}$, respectively. Increasing $\beta$ and so perturbing away from the Lorenz equations has not only increased the periods of the upos, but has eliminated some of those present in the Lorenz limit, such as $L_{51}$ that we observed in our benchmark integrations. Of course, there is nothing to prevent such orbits appearing during a longer integration or for a different initial data, but that was not our objective here.

Increasing $\beta$ to $\beta=2$, Figure 9 shows the growing prominence of the two-banded structure of the first return maps; Figure 10 shows the corresponding histogram. Immediately apparent is the increase in the number of $L_{11}$ orbits, as well as the relatively clean peaks at other periods. Figure 11 shows part of the time series for $Y(t)$, together with a selection of phase portraits, obtained using the same initial data. In Figure 12 we show the only $L_{n 1}$-series upos which are present in the histogram. These are the $L_{11}$ orbit with period $1.591 \mathrm{~s}$, the $L_{21}$ orbit (period $2.417 \mathrm{~s}$ ) and the $L_{31}$ orbit (period $3.239 \mathrm{~s}$ ). A selection of the lowest order $L_{n 2}$-series orbits are shown in Figure 13, including one which involves 


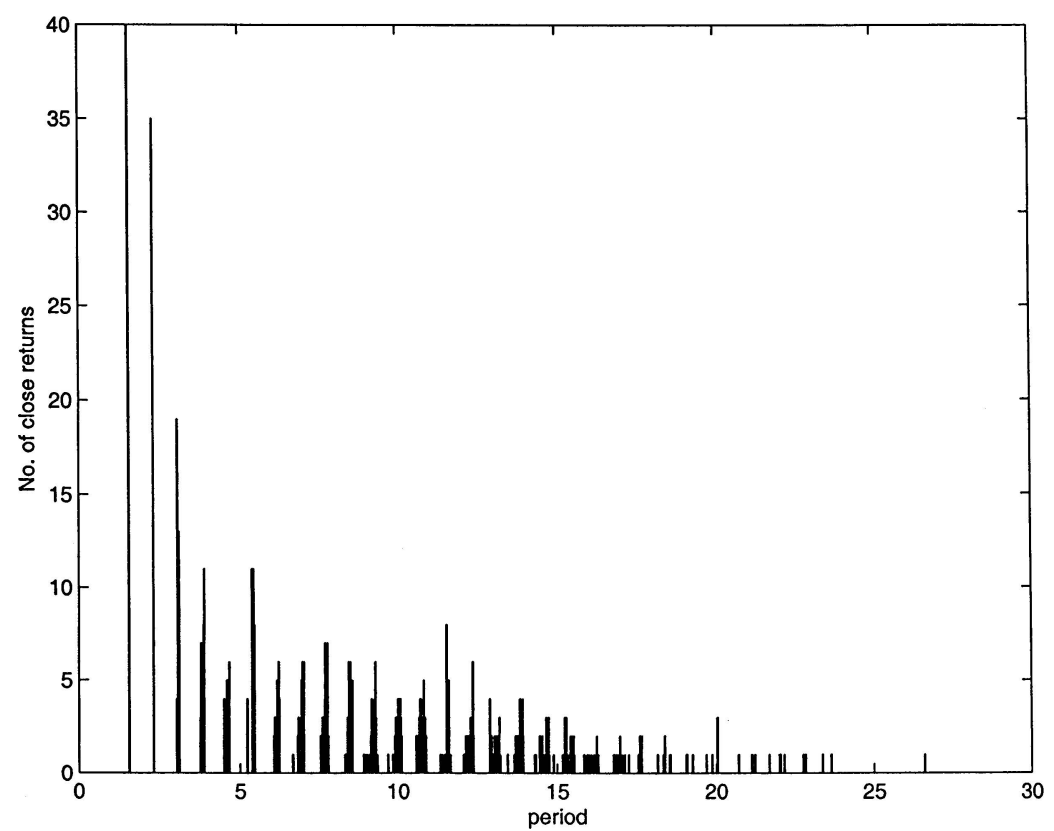

Figure 8. The histogram of close returns up to and including the 10th iterates for $\Lambda=3.2$ and $\beta=0.5$.
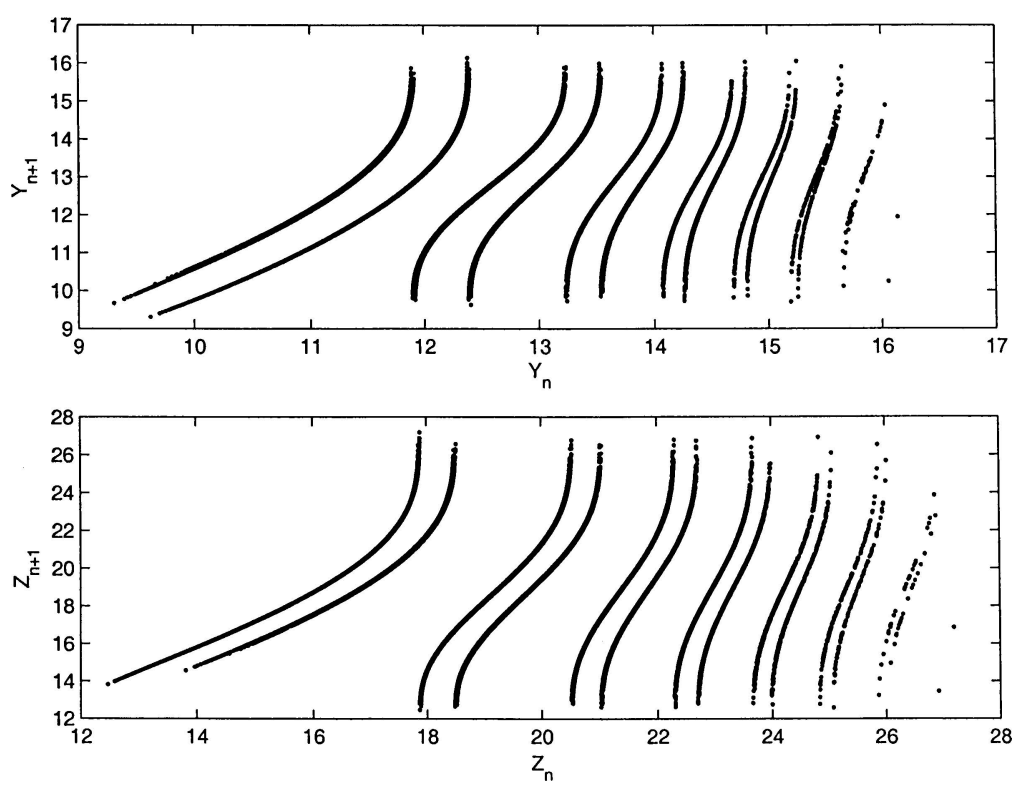

Figure 9. As in Figure 7 but for $\beta=2$.

a different ordering of the circuits around the two nontrivial equilibrium states. They are $L_{22}$ (period $3.256 \mathrm{~s}$ ), $L_{32}$ (periods 4.081 and $4.012 \mathrm{~s}$, respectively) and $L_{52}$ (period of $5.748 \mathrm{~s}$ ). We remark that the upo, which cycles twice around one of the equilibria and then three times around the other, has a slightly longer period than its companion. Again the values of the periods of the upos are larger than their Lorenz counterparts and for the $\beta=2$ case. 


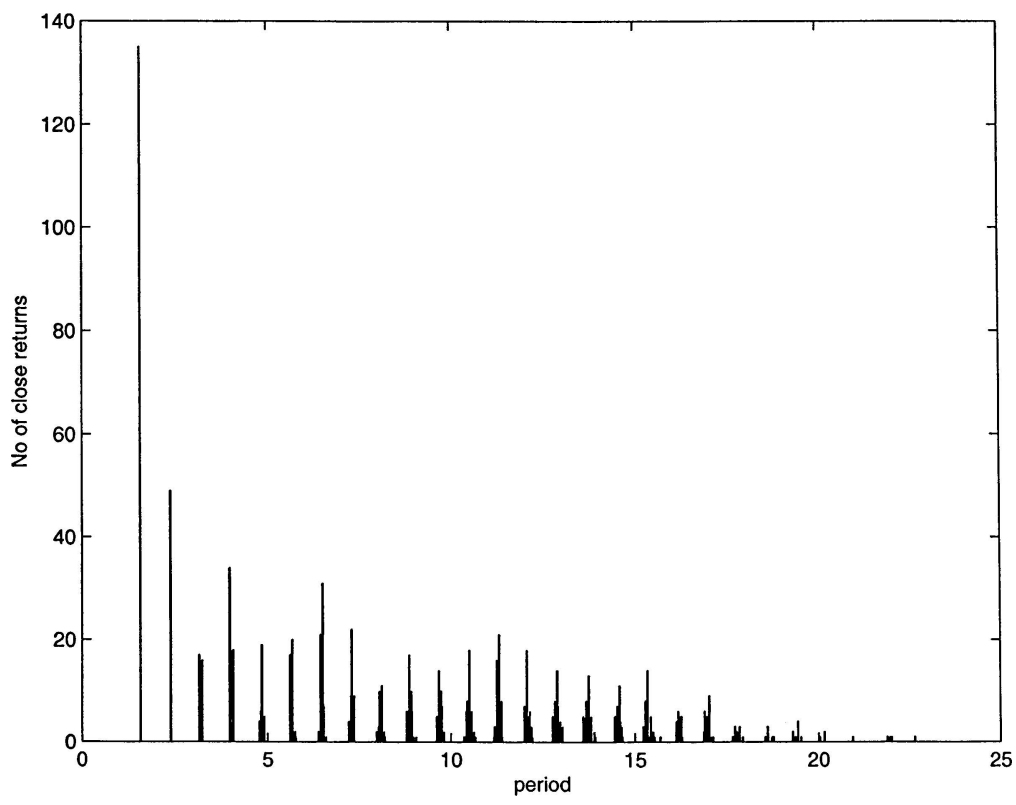

Figure 10. As in Figure 8 but for $\beta=2$.
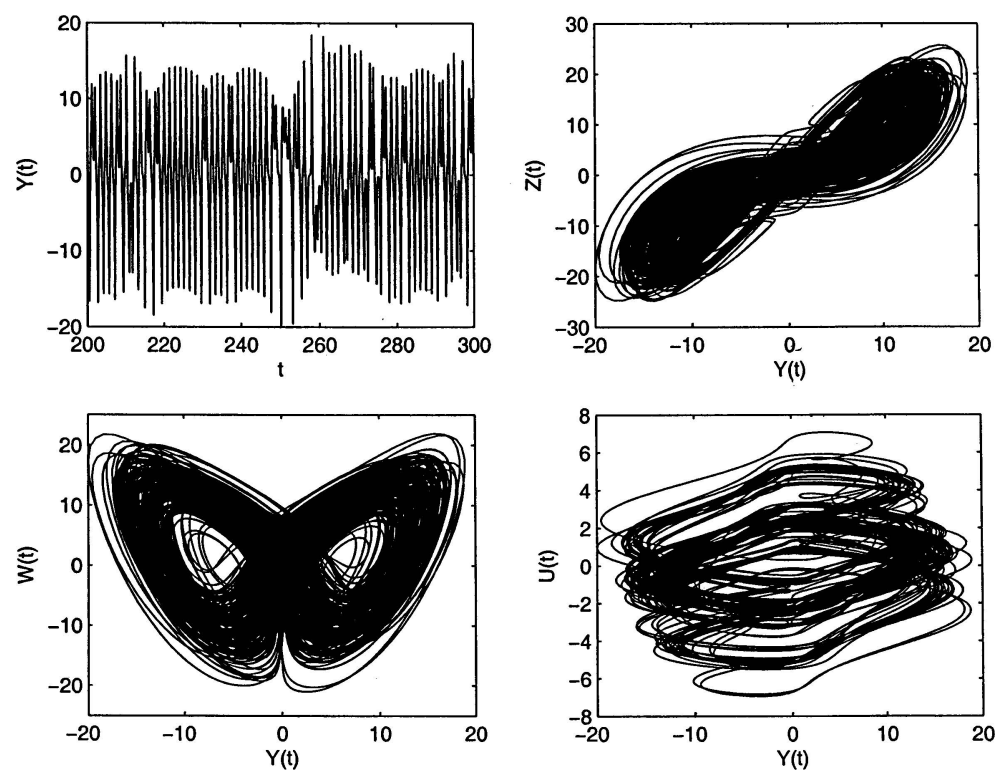

Figure 11. A section of the time series of $Y(t)$, together with a selection of phase portraits for $\beta=2$.

Our final illustration in this subsection is for $\beta=7.25$, close to the loss of chaos and transition to steady dynamo action. The first return maps (Figure 14) now show that many of the upos have disappeared and the double banding structure is converging in the $\left(Y_{n}, Y_{n+1}\right)$ plot. The $L_{11}$ upo now dominates the histogram (Figure 15). The only series $L_{n 1}$ upos observed in our integrations for $\beta=7.25$ are the $L_{11}$ orbit with period $1.967 \mathrm{~s}$ and the $L_{21}$ orbit with period $3.104 \mathrm{~s}$. These are shown in Figure 16, while Figure 17 shows the lowest order $L_{n 2}$ cycles: the $L_{22}$ (period $4.152 \mathrm{~s}$ ) and two $L_{32}$ cycles (periods 5.295 and 5.063 s, respectively). Finally Figure 18 shows the actual time series and phase portraits. 

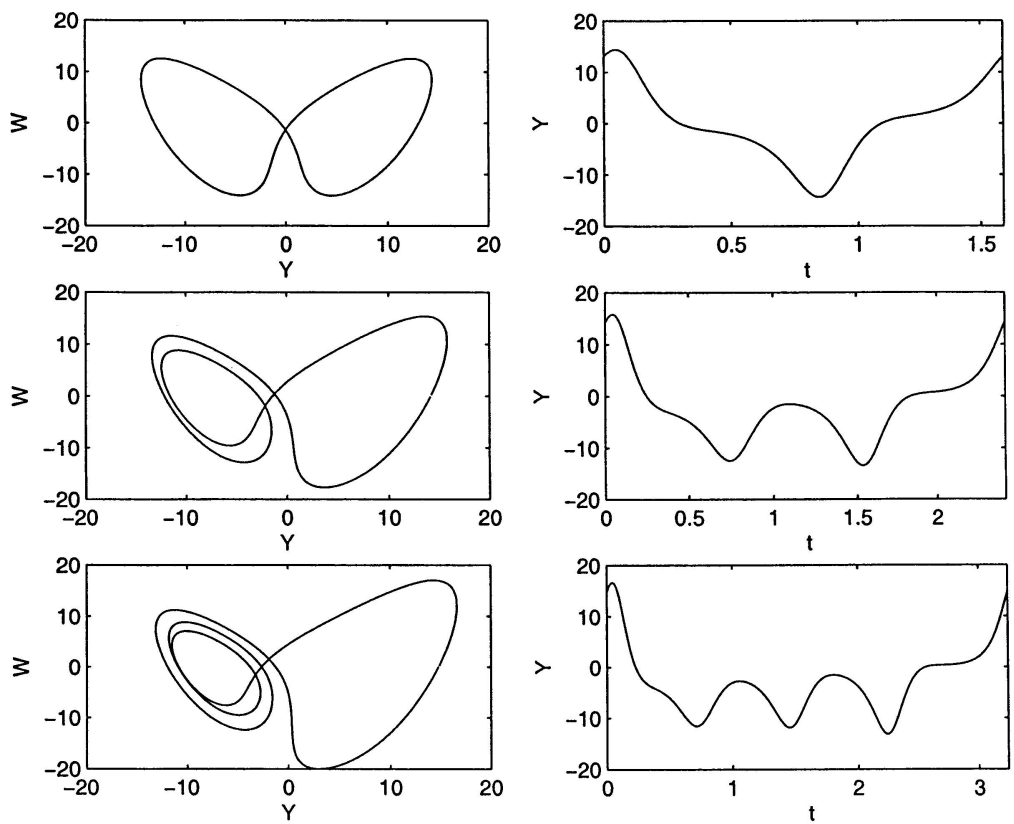

Figure 12. The series $L_{n 1}$ present in the histogram of Figure 10.
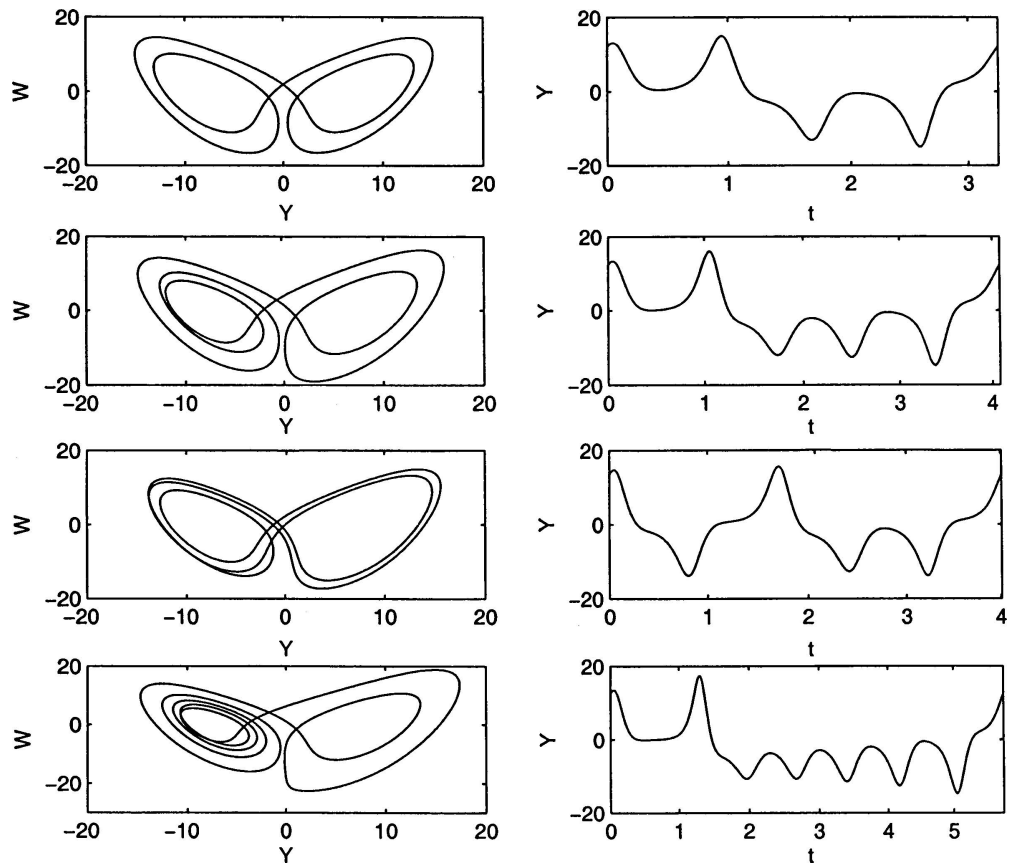

Figure 13. Some of the lowest order series $L_{n 2}$ present in the histogram of Figure 10.

\section{2. $\Lambda<1$ AND $\sigma>1+v$}

When $\Lambda=0.5$, Figure 6 shows that the loss of chaotic behaviour, arising from the $\beta=0$ limit, is now to stable periodic dynamo action for $\beta \approx 4$. This periodic behaviour is associated with a supercritical 

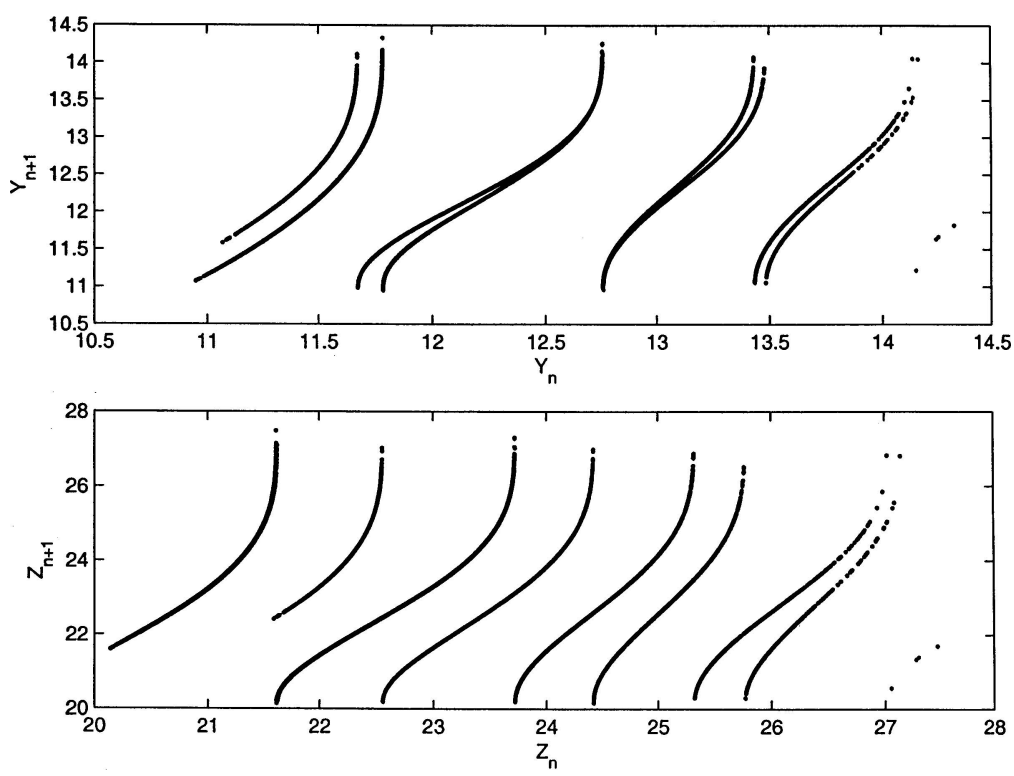

Figure 14. As in Figure 7 but for $\beta=7.25$.

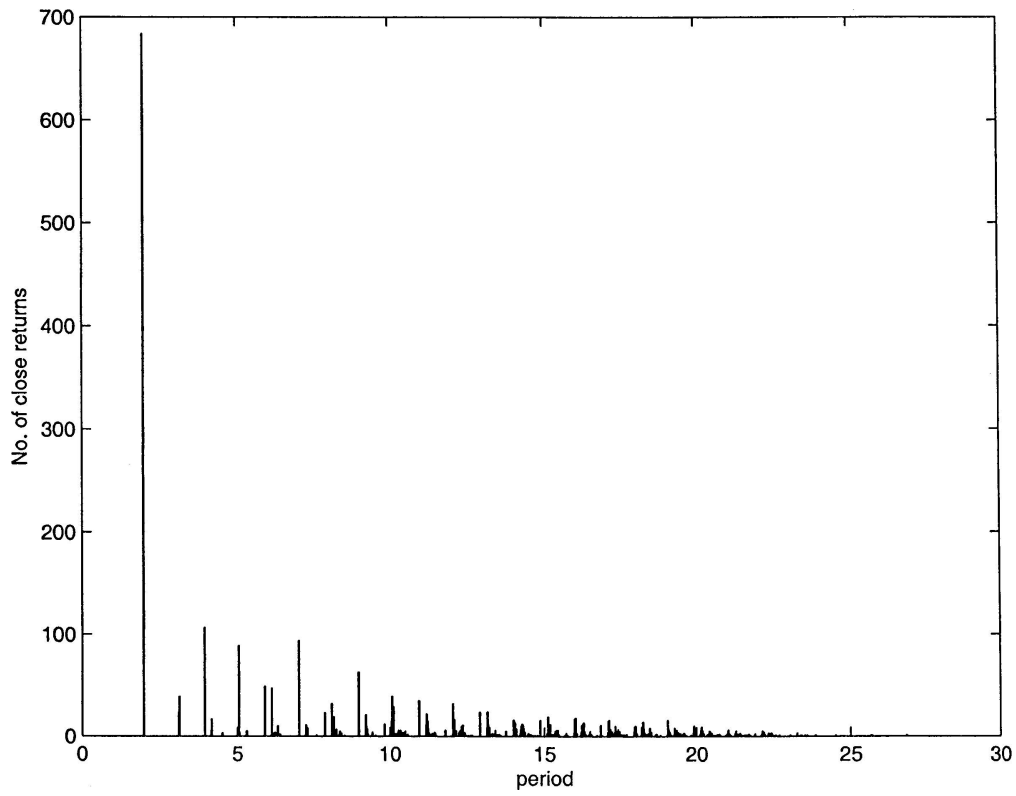

Figure 15. As in Figure 8 but for $\beta=7.25$.

Hope bifurcation [3] from the trivial equilibrium state (20) and results in radically different appearances of the first return maps and the histograms of upos. The presence of the Takes-Bodansky double-zero bifurcation from which emanate a supercritical Hope bifurcation (associated with the trivial equilibrium state) and a subcritical Hope bifurcation (associated with the nontrivial equilibria), terminating at the Lorenz subcritical Hope bifurcation point, strongly influence the upo spectrum and render the $\Lambda<1$ case far more sensitive to perturbations from $\beta=0$ than when $\Lambda>1$. 

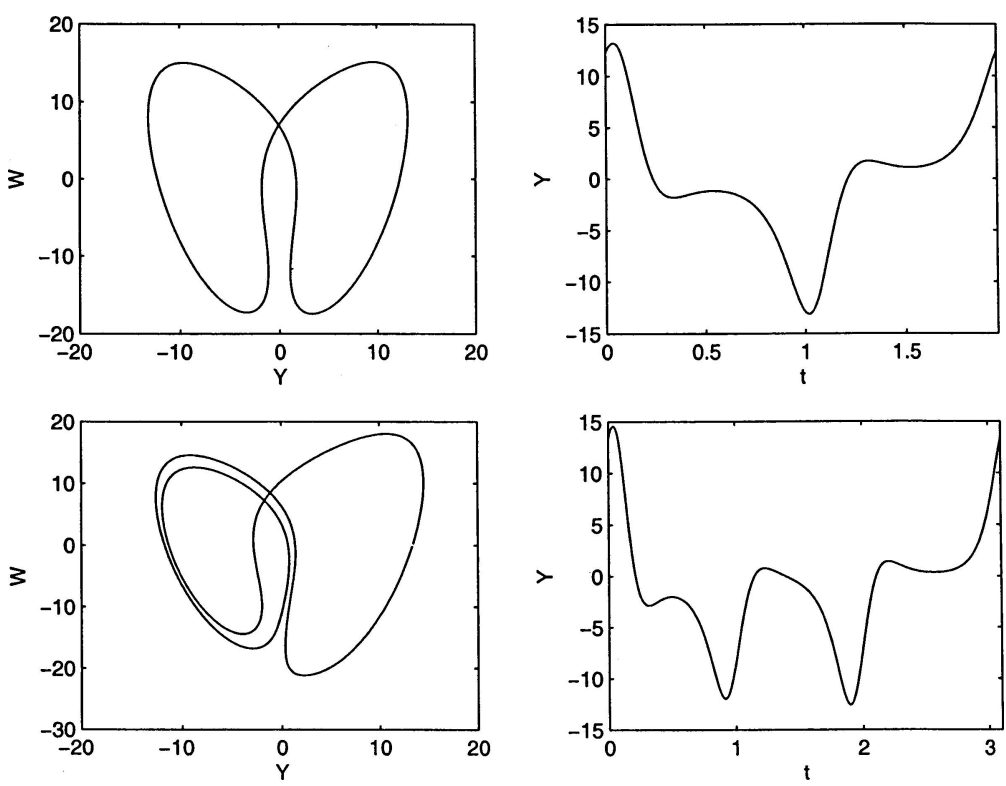

Figure 16. The only $L_{n 1}$ present in the histogram of Figure 15.
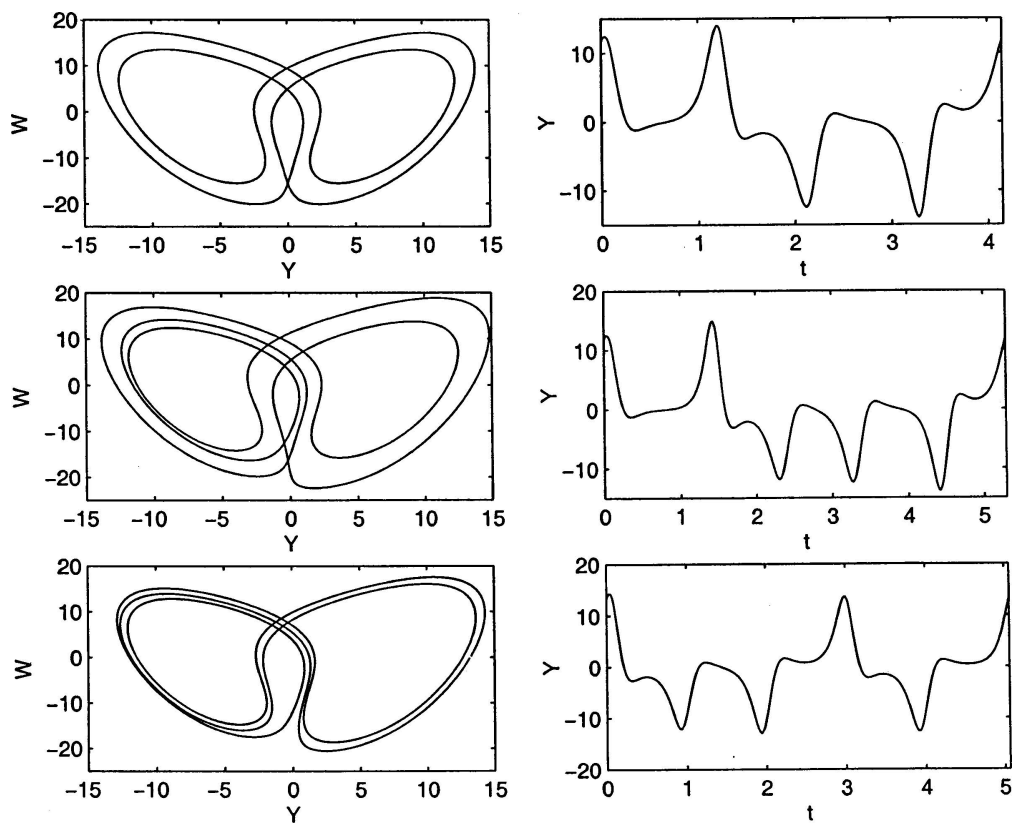

Figure 17. The lowest order series $L_{n 2}$ observed in the histogram of Figure 15.

Figure 19 shows the first returns maps in $\left(Y_{n}, Y_{n+1}\right)$-space for $\beta=0.01, \beta=0.02, \beta=0.04$ and $\beta=0.08$. The corresponding histograms, not shown here, contain very few points. For example, when $\beta=0.5$, we only found 9 close returns with $60,000,000$ time steps. Gone is the elegant system of curves obtained by Koga [13] for the $\left(Y_{n}, Y_{n+1}\right)$ plots, while the $\left(Z_{n}, Z_{n+1}\right)$ plots (not shown here) still retain traces of that structure. The situation improves, however, as we increase $\beta$. 

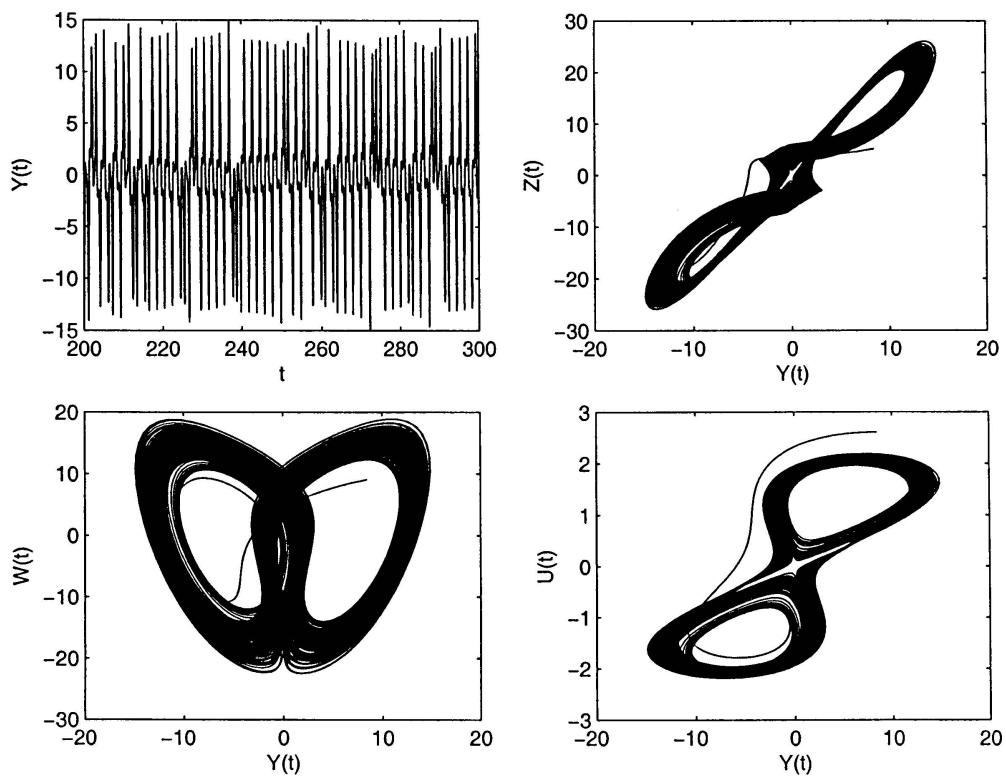

Figure 18. A section of the time series of $Y(t)$, together with a selection of phase portraits for $\beta=7.25$.
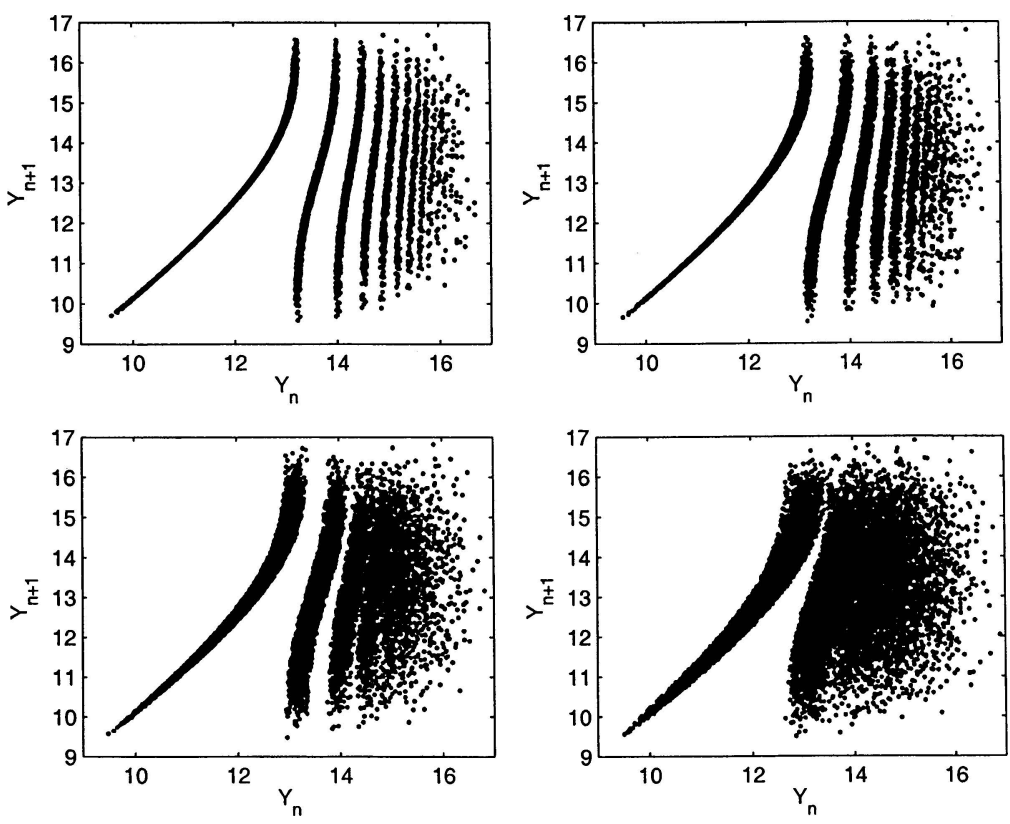

Figure 19. The first return maps in the $\left(Y_{n}, Y_{n+1}\right)$-plane for $\Lambda=0.5$ and $\beta=0.01, \beta=0.02, \beta=0.04$ and $\beta=0.08$.

Figure 20 shows the first return maps, while Figure 21 shows the histogram for $\beta=2$. The only series $L_{n 1}$ upo observed was $L_{11}$ with period $1.442 \mathrm{~s}$ and shown as the top pair in Figure 22. We remark that this period is lower than the corresponding $L_{11}$ of the Lorenz equations (whose period is $1.5587 \mathrm{~s}$ ).

Structure begins to re-emerge in the return maps when $\beta$ is increased further, while the histograms get much simpler. Figures 23 and 24 show the scenario for $\beta=3.5$. The upo $L_{11}$ dominates with a 

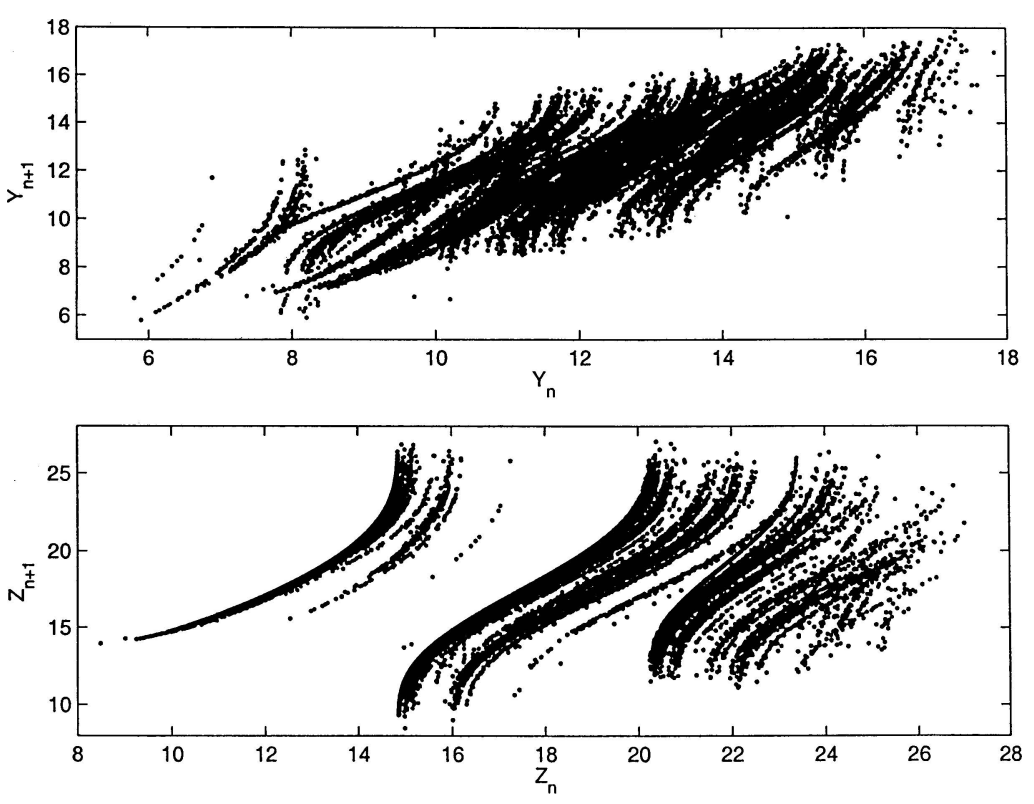

Figure 20. As in Figure 9 but for $\Lambda=0.5$.

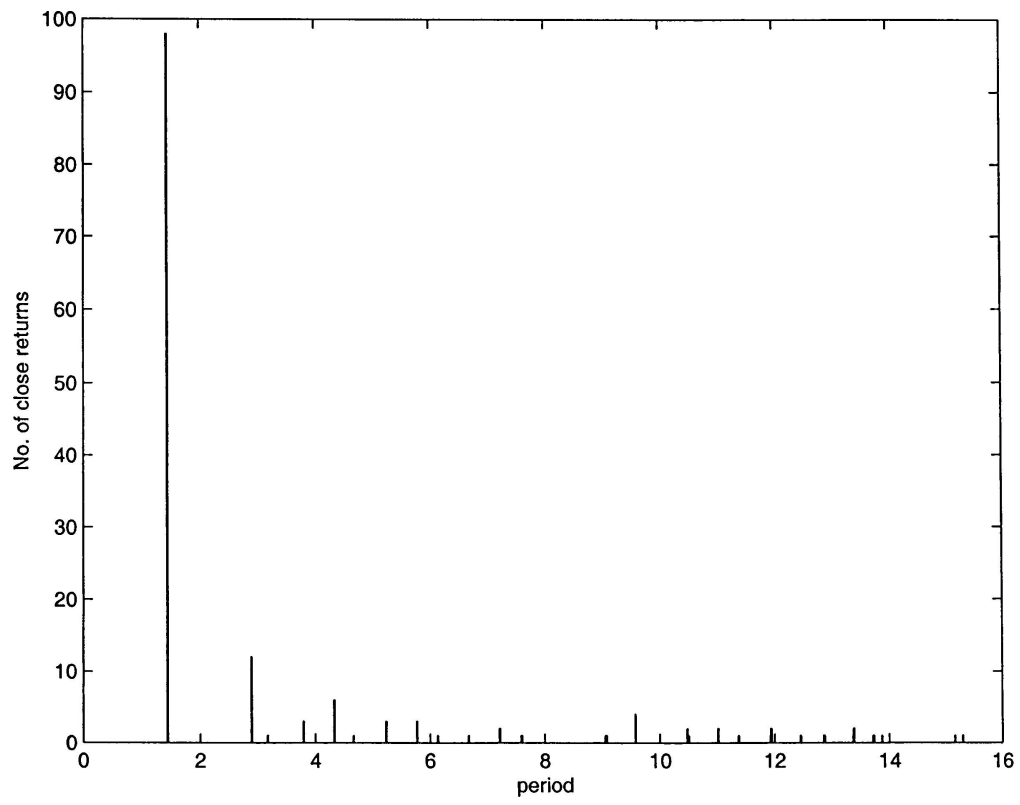

Figure 21. As in Figure 10 but for $\Lambda=0.5$.

period of $1.389 \mathrm{~s}$, reflecting the growing influence of the stable limit cycle, which is the only feature of the dynamics for $\beta>4$.0. Figure 25 shows the phase portraits in the $(Y, \mathcal{W})$-plane for $\beta=0.01$, $\beta=3.5, \beta=4.0$ and $\beta=4.1$. Although the plot for $\beta=0.01$ has the nontrivial equilibria passing through $\mathcal{W}=0$, this is clearly not the case for $\beta=4$.1. Indeed the other two phase portraits show a competition in play between these two limiting influences. 

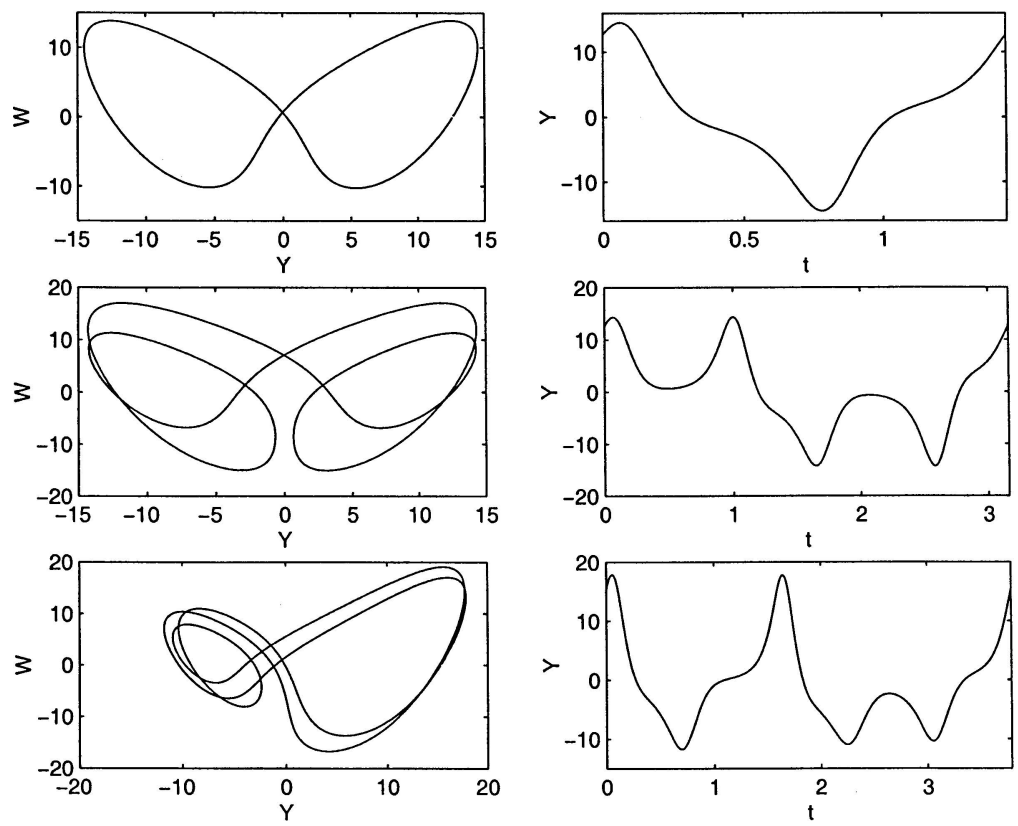

Figure 22. As in Figure 12 but for $\Lambda=0.5$.
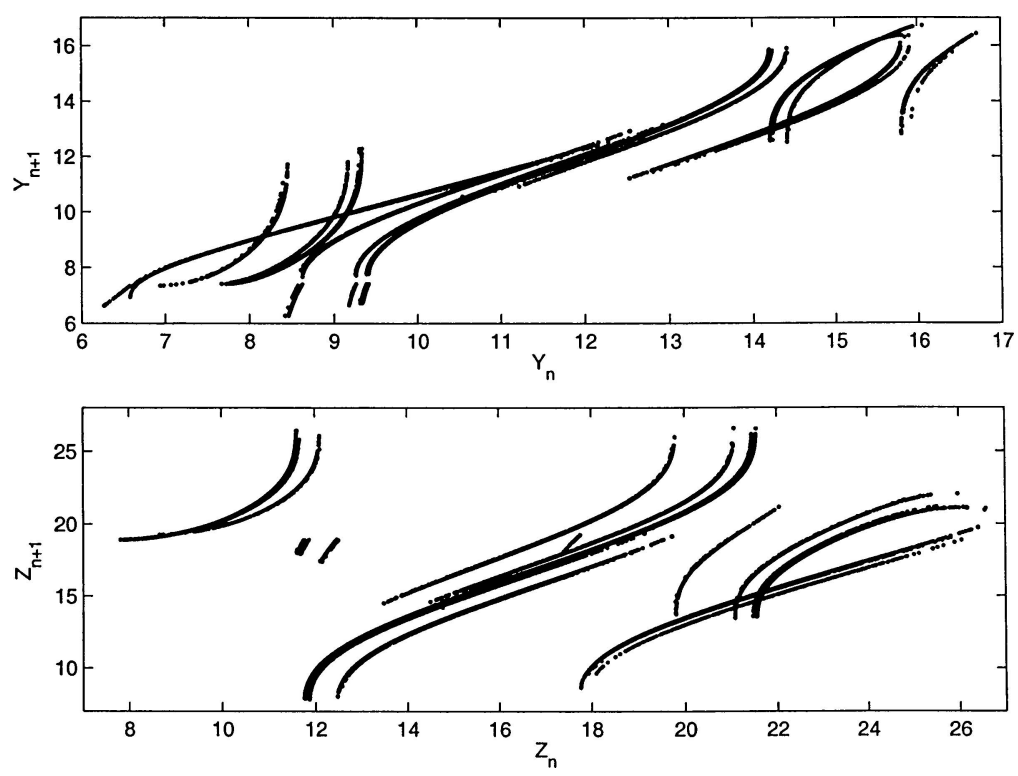

Figure 23. As in Figure 20 but for $\beta=3.5$.

\section{Discussion}

The importance of self-exciting Faraday disk dynamos, of which the EMR dynamo is one example, rests in their containing some of the key ingredients of the large-scale naturally occurring MHD dynamos, while being of considerably lower dimension and so much more amenable to systematic investigations. 


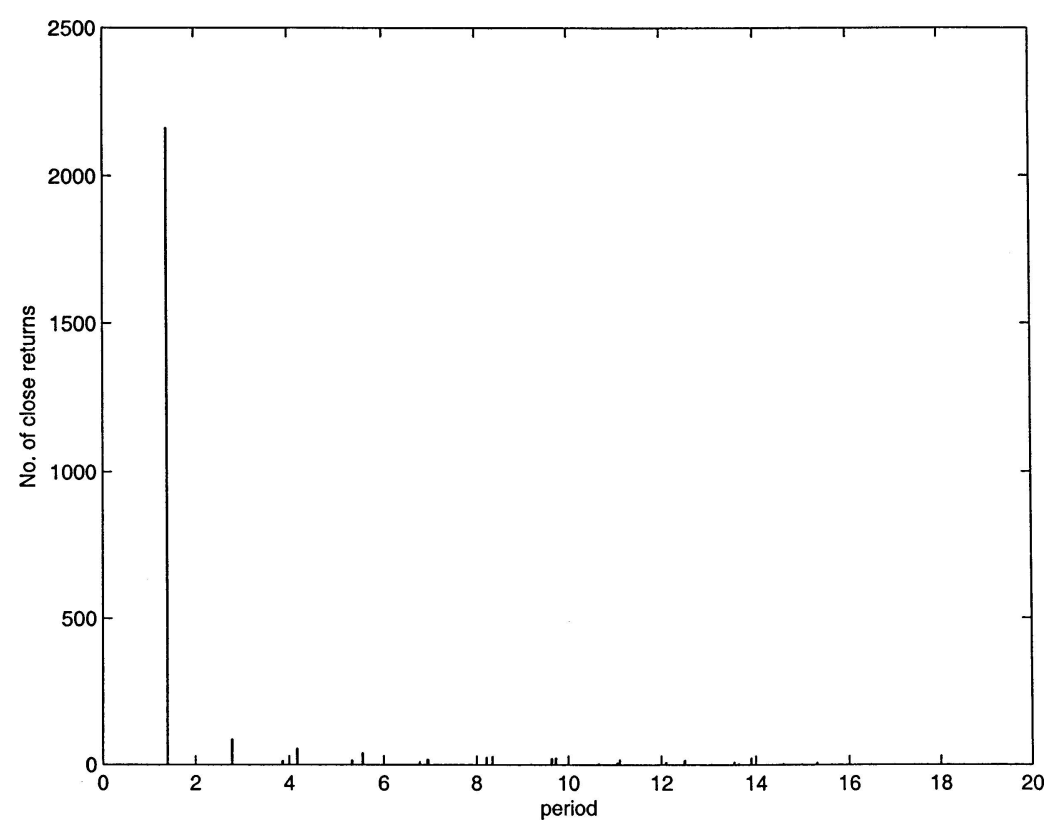

Figure 24. As in Figure 21 but for $\beta=3.5$.
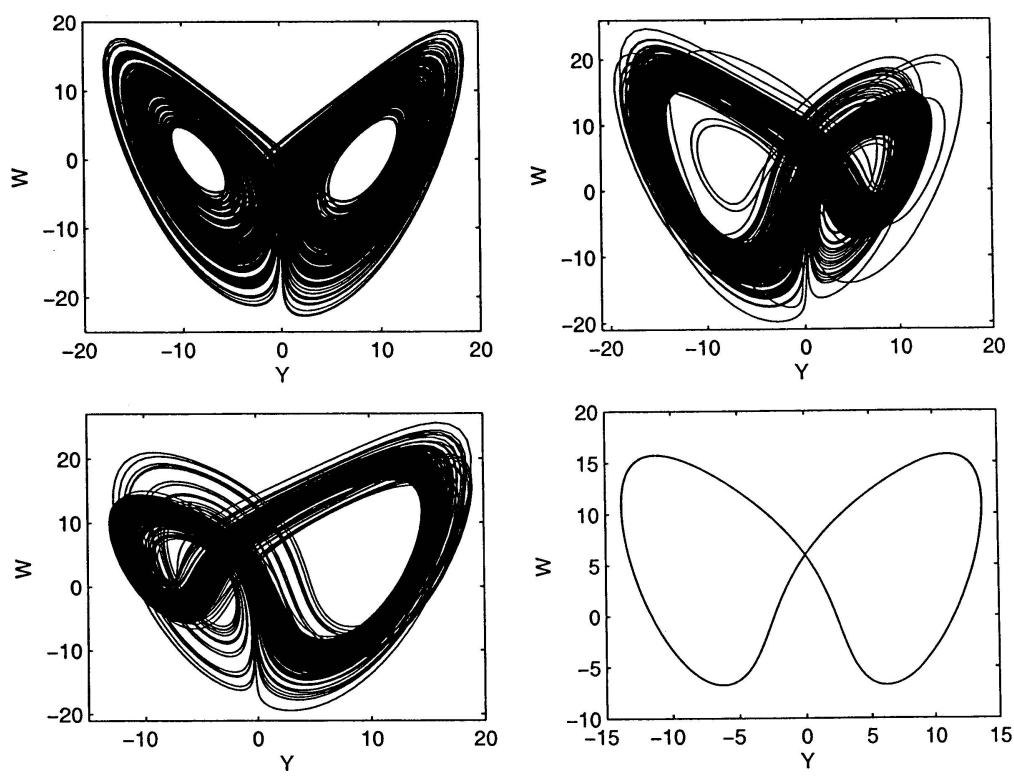

Figure 25. The phase portraits in the $(Y, \mathcal{W})$-plane for $\beta=0.01, \beta=3.5, \beta=4.0$ and $\beta=4.1$.

They can exhibit irregular reversals, as well as steady dynamo action, multiple solutions (due to hysteresis effects) and the nonlinear quenching of oscillatory behaviour [19].

Since so many of the low order dynamo models of this family, investigated since [5], have rich ranges of behaviour with reversals a feature, what has been lacking has been some way of distinguishing between all of these and other models as a prelude to comparing them with the large-scale counterparts. 
One possible avenue is via the identification of the spectrum of unstable periodic orbits, as well as their behaviour when key parameters in the problem vary.

A first attempt along these lines has been achieved with the present study for the EMR dynamo. This system has the added interest of reducing to the Lorenz equations when one of the parameters vanishes. Since these equations have received considerable attention in the literature with regard to their upo structure, perturbations away from the Lorenz limit were viewed as an ideal starting point. Future work will build upon this as well as investigating two of the other Faraday disk dynamos that have the Lorenz equations as a limiting case $[2,4]$. Others do not and their upo structure has yet to be determined; these will also be investigated.

\section{References}

1. Hide, R. and Moroz, I. M., 'Effects due to induced azimuthal eddy currents in the self-exciting Faraday disk homopolar dynamo with a nonlinear series motor. Part I . Two special cases', Physica D 134, 1999, 387-301.

2. Moroz, I. M. and Hide, R., 'Effects due to induced azimuthal eddy currents in the Faraday disk self-exciting homopolar dynamo with a nonlinear series motor. Part II. The general case', International Journal of Bifurcation and Chaos 2000 , 2701-2716.

3. Moroz, I. M., 'The Malkus-Robbins dynamo with a linear series motor', International Journal of Bifurcation and Chaos 13, 2003, 147-161.

4. Moroz, I. M., 'The Malkus-Robbins dynamo with a nonlinear series motor', International Journal of Bifurcation and Chaos 14, 2004, 2885-2892.

5. Hide, R., Skeldon, A. C., and Acheson, D. J., 'A study of two novel self-exciting single-disk homopolar dynamos: Theory', Proceedings of the Royal Society of London A 452, 1996, 1369-1395.

6. Moroz, I. M., 'On the behaviour of a self-exciting Faraday-disk homopolar dynamo with a variable nonlinear series motor', International Journal of Bifurcation and Chaos, 2002, 2123-2135.

7. Moroz, I. M., Hide, R., and Soward, A. M., 'On self-exciting coupled Faraday disk homopolar dynamos driving series motors', Physica D 117, 1998, 128-144.

8. Hide, R., 'The nonlinear differential equations governing a hierarchy of self-exciting coupled Faraday-disk homopolar dynamos', Physics of the Earth and Planetary Interiors 103, 1997, 281-291.

9. Moroz, I. M., Smith, L. A., and Hide, R., 'Synchronised chaos in coupled double disk homopolar dynamos', International Journal of Bifurcation and Chaos 8, 1998, 2125-2133.

10. Moroz, I. M., 'Synchronised dynamics in three coupled Faraday disk homopolar dynamos', in Fluid Dynamics and the Environment: Dynamical Approaches, Springer Verlag (ed.), 2000, pp. 225-238.

11. Goldbrum, P., Moroz, I. M., and Hide, R., 'On the biasing effect of a battery on a self-exciting Faraday disk homopolar dynamo loaded with a linear series motor', International Journal of Bifurcation and Chaos 10, 2000, $1875-1885$.

12. Moroz, I. M., 'Behaviour of a self-exciting Faraday-disk homopolar dynamo with battery in the presence of an external magnetic field', International Journal of Bifurcation and Chaos, 2001, 1695-1705.

13. Koga, S., 'Phase description method to time averages in the Lorenz system', Progress of Theoretical Physics 76, 1986, 335-355.

14. Carroll, T. L., 'Approximating chaotic time series through unstable periodic orbits', Physical Review E 59, $1999,1615-1621$.

15. Robbins, K. A., 'A new approach to sub-critical instability and turbulent transitions in a simple dynamo', Mathematical Proceedings of the Cambridge Philosophical Society 82, 1977, 309-325.

16. Hénon, M., 'On the numerical computation of Poincaré maps', Physica D 5, 1982, 412-414.

17. Franceschini, V., Gilberti, C., and Zheng, Z., 'Characterisation of the Lorenz attractor by unstable periodic orbits', Nonlinearity 6, 1993, 251-258.

18. Viswanath, D., 'Symbolic dynamics and periodic orbits of the Lorenz attractor', Nonlinearity 16, 2003, $1035-1056$.

19. Hide, R. and Moroz, I. M., 'Physically realistic Faraday disk dynamos', in Mathematical Aspects of Natural Dynamos, Springer-Verlag (ed.), 2004. 